\title{
Articulación de sistemas de gestión HSEQ e ISO/IEC 17025:2005 aplicables en los laboratorios de calibración acreditados en maǵnitudes químicas en Bogotat
}

\section{Articulation of ESHQ and ISO/IEC 17025:2005 management systems applicable to accredited calibration laboratories with chemical magnitudes in Bogotá}

Nelcy Yamile Ortega Gómes"

Organismo Nacional de Acreditación de Colombia - ONAC

\section{RESUMEN}

Considerando la importancia que tienen actualmente los sistemas de gestión HSEQ en las organizaciones que prestan servicios en la industria, y que Colombia se abre a nuevos mercados internacionales a través de la aplicación de la norma ISO/IEG 17025:2005, se identifica la necesidad de conocer la articulación entre la acreditación, fundamentada en la norma ISO/ IEC17025 y la certificación del sistema de gestión HSEQ; esto es particularmente significativo en los laboratorios de calibración para magnitudes químicas por la importancia que tienen para la industria. Este estudio consideró una muestra de laboratorios de calibración para magnitudes químicas que están acreditados se consultó la base de datos del Organismo Nacional

Artículo de resultado de investigación.

** Química Industrial. Candidata a magíster en Calidad y Gestión Integral del Convenio Interinstitucional USTA - Icontec. Correo electrónico: nelcy_83@yahoo.com 
de Acreditación de Colombia en el mes de abril del año 2014; se utilizó como instrumento de recolección de datos una encuesta dirigida a los representantes del sistema de gestión y la alta dirección. Esta encuesta fue validada por expertos y sometida a una prueba piloto. Se identificaron aspectos de los sistemas de gestión enmarcados en el ciclo PHVA para medir la optimización de recursos en términos de eficacia (resultados) y eficiencia (procesos) en estos laboratorios.

Palabras clave: globalización, subsistema nacional de la calidad, acreditación, certificación, ISO, sistemas de gestión integrados.

\section{ABSTRACT}

Viewing current importance ESHQ management systems in organizations providing services in the industry, and today Colombia opens new international markets with the implementation of ISO/IEC 17025:2005, is the need to know and identify the articulating elements between accreditation and certification, so that will strengthen the quality of services provided by calibration laboratories are accredited chemical quantities. And these services are performed by competent personnel, qualified, motivated to develop labor activities in decent working conditions that provide and deploy environmental management in order to achieve customer loyalty and new business opportunities in a globalized Colombia which has internationally recognized. For this reason, we have developed this study: "Articulation of ESHQ management systems and ISO/IEC 17025:2005 applicable to calibration laboratories accredited chemical quantities in Bogotá". To determine the sample of accredited laboratories database of the National Accreditation Agency of Colombia in April 2014 were consulted; was used as data collection instrument for the structured survey management system representatives and senior management, which was validated by experts and subjected to a pilot. Aspects of management systems framed in the PDCA cycle to measure the value for money in terms of effectiveness (results) and efficiency (processes) in these laboratories were identified.

Keywords: Globalization, national quality subsystem, accreditation, certification, ISO, integrated management system.

\section{INTRODUCCIÓN}

Partiendo del hecho que la certificación (NTC-ISO 9001:2008. Sistema de gestión de la calidad. Requisitos) es la atestación de tercera parte relativa a productos, procesos, sistemas o personas, y que la acreditación (NTC-ISO/IEC17025:2005. Requisitos generales para la competencia de los laboratorios de ensayo y calibración) es la atestación de tercera parte relativa a un organismo de evaluación de la conformidad que manifiesta la demostración formal de su competencia para llevar a cabo tareas específicas de evaluación de la conformidad; y en atención a las iniciativas del gobierno, la industria y la academia con miras hacia un mundo globalizado, es importante conocer en el contexto nacional la interacción de los Sistemas de Gestión Integral HSEQ en cuanto a la certificación de sus procesos versus la acreditación bajo la ISO/IEC 17025:2005 de los laboratorios de calibración que prestan servicios para un alcance en magnitudes químicas de $\mathrm{pH}$ (potencial de hidrógeno) y análisis químicos. Teniendo en cuenta las fortalezas y garantías de competitividad adquiridas por estos laboratorios acreditados, que han permitido a su vez fortalecer y garantizar la competitividad técnica a nivel nacional; y a hoy día surge una inquietud frente a un reconocimiento internacional para cubrir diversos aspectos en la consecución de los objetivos propuestos 
por los programas nacionales para el fomento de la competitividad y productividad (Superintendencia de Industria y Comercio, 2006).

El gobierno da las pautas, la industria la necesidad y la academia la oportunidad de proporcionar soluciones 0 mejoras en esta interacción. Esta es una de las razones del desarrollo de esta investigación, ya que conduce a la articulación entre los sistemas de certificación HSEQ enmarcados en el campo de la acreditación a través de una muestra representativa del sector de laboratorios de calibración acreditados en magnitudes químicas ubicados en la ciudad de Bogotá, y cuyo resultado será una propuesta de requerimientos orientados a fortalecer su competencia con aspectos ambientales y de salud ocupacional relevantes para estos organismos evaluadores de la conformidad y de esta manera generar resultados eficaces y eficientes enmarcados en Sistemas de Gestión Integrados.

\section{METODOLOGÍA}

En la figura 1, se describen las cuatro fases que se llevaron a cabo para el desarrollo del proyecto, el cual se clasifica como una investigación aplicada, explicativa de carácter mixto, es decir cuali-cuantitativo (Cerda, 2011, p. 79), ya que permite conocer los ítems articulados y las relaciones entre las variables en el sistema de gestión para cada una de las normas a trabajar durante el desarrollo de esta investigación.

Figura 1. Fases de la investigación.

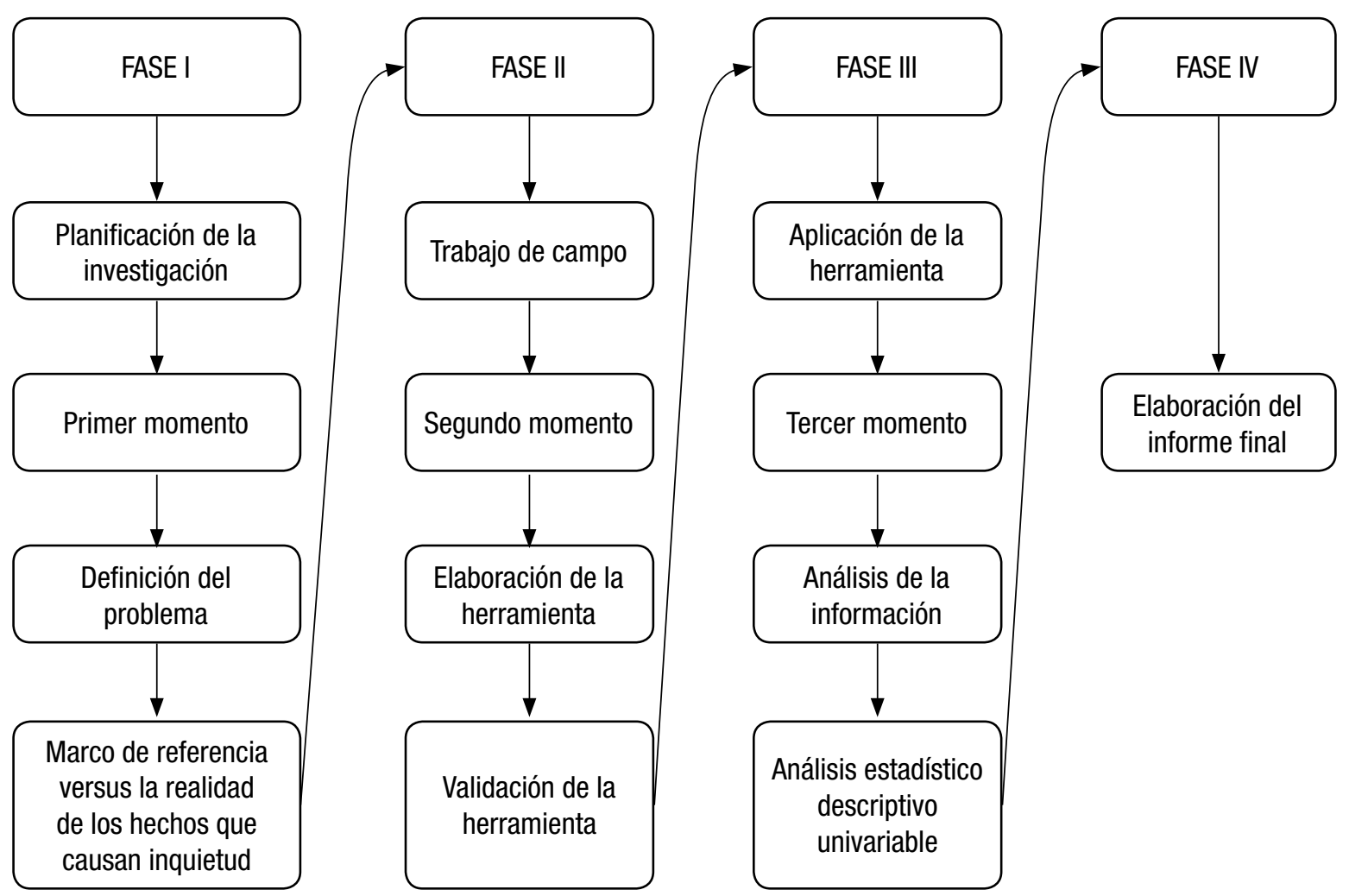

Fuente: elaboración propia. 
Los criterios para validar la información documental en los cuales se basó esta investigación salieron de los requisitos establecidos en cada una de las normas de certificación y acreditación.

La representatividad de la muestra se soporta en el directorio oficial de laboratorios de calibración acreditados publicado en la página web de la ONAC, y la confiabilidad está determinada por la construcción de los instrumentos de recolección de datos basados en encuestas validadas y realizadas a toda la población, con el fin de determinar los efectos de los sistemas de gestión en la eficacia y eficiencia, y de esta manera conocer, identificar, consolidar e interpretar la información relevante de estos organismos evaluadores de la conformidad; razón por la cual se define como tipo de muestreo poblacional y como método censal.

\section{RESULTADOS Y DISCUSIÓN}

La información obtenida durante el desarrollo de esta investigación es del total de la población porque los datos obtenidos son de los 6 laboratorios de calibración acreditados en magnitudes químicas en la ciudad de Bogotá, cuyos elementos de esta población permiten realizar estadística descriptiva univariada (Canavos, 1988).

Se espera con la implementación de esta matriz favorecer la competitividad y productividad de los laboratorios de calibración, toda vez que se promueve la eficacia y optimización de recursos como resultado de la implementación de un sistema de gestión integrado que permita el fortalecimiento de su competencia técnica, consolidando requisitos articuladores en los laboratorios de calibración acreditados en magnitudes químicas y ubicados en la ciudad de Bogotá.

\section{ARTICULACIÓN DE LOS SISTEMAS DE GESTIÓN}

A continuación, en la figura 2, se presenta la matriz teórica de articulación entre las normas de acreditación y certificación, teniendo como premisa que los laboratorios de calibración en magnitudes químicas están acreditados bajo un sistema de gestión NTC-ISO/IEC 17025:2005, y considerando que en estos no se tiene un sistema de gestión integrado en las normas NTCISO 9001:2008, NTC-OHSAS 18001:2007, NTC-ISO 14001:2004.

Se parte desde el componente técnico, es decir, la competencia técnica del personal para la ejecución de las calibraciones con una mirada de la integralidad hacia los sistemas de gestión enmarcados en el Subsistema Nacional de la Calidad (Decreto 2269, 1993), teniendo en cuenta el contexto en el cual se desempeña la organización que para este caso se denominan Organismos Evaluadores de la Conformidad - OEC (NTC-ISO/ IEC17011:2005).

Figura 2. Articulación del componente técnico versus sistema de gestión.

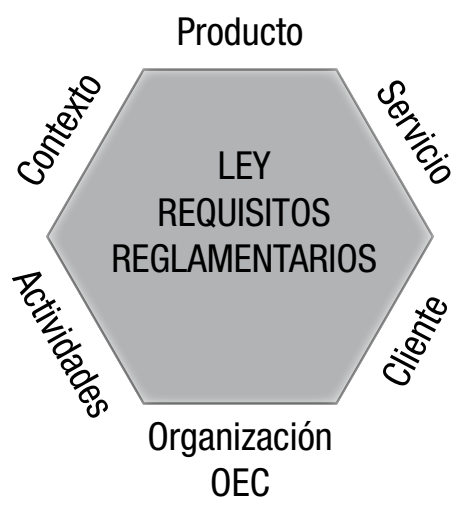

Fuente: elaboración propia (2014-09-05).

Los laboratorios acreditados tienen implementado un sistema de gestión de la Norma ISO/IEC 17025:2005 cuyos requisitos estructurales del 5.2 al 5.10 están relacionados con la formación, información, comunicación 
y competencia técnica, áreas que van ligadas a la innovación y aprendizaje.

Respecto a la ISO 9001:2008, cuyo enfoque es hacia clientes fidelizados mediante la calidad de procesos productos y servicios; los requisitos de interacción son los que corresponden a 5.1, 5.2, 5.5, 6.1, 6.2, 6.3 y 7.1, 7.2.1, 7.2.2, 7.3, 7.4, 7.5, aquellos del sistema de gestión de certificación que son comunes con el sistema de gestión en acreditación.

Para la ISO 14001:2004 (Sistemas de gestión ambiental. Requisitos con orientación para su uso) los requisitos de interacción son 4.3.1, 4.3.2, 4.4.1, 4.4.2, 4.4.6 y 4.5.1, 4.5.2, 4.5.3, aquellos que permiten una mirada hacia una sociedad favorecida, donde el laboratorio de calibración acreditado en magnitudes químicas demuestre una calidad medioambiental por la gestión de los residuos que maneja tanto en las instalaciones fijas como en las instalaciones de sus clientes.

Para los laboratorios acreditados con implementación en OSHAS 18001:2007 (Sistemas de gestión en seguridad y salud ocupacional), los requisitos de interacción son 4.3.1, 4.3.2, 4.4.1, 4.4.2, 4.4.6, 4.5.1, 4.5.2, 4.5.3, que cuentan con personal, además de calificado, motivado en condiciones de trabajo digno.

En la tabla 1 se observa la correspondencia de interacción entre normas de un sistema de gestión de calidad acreditable y certificable de acuerdo con sus requisitos de interacción que permiten unificar, consolidar y hacer más eficaz las actividades a desarrollar para cada uno de los procesos donde se puede complementar como lista cruzada con los documentos del sistema de gestión de cada OEC.

Esta correspondencia de interacción sirvió de base para el bosquejo de la articulación del sistema de gestión integrado, según la figura 3. La cual surge del mismo planteamiento de articulación existente en el país en cuanto al Subsistema Nacional de Calidad (normalización, acreditación y metrología), donde el centro o fundamento de esta interacción son los organismos evaluadores de la conformidad - OEC. Así mismo, se quiso plantear la interacción de los sistemas de gestión de calidad entre certificación y acreditación teniendo como fundamento central la innovación y aprendizaje del personal que labore en condiciones de trabajo dignas con calidad medioambiental para la prestación de un servicio.

Figura 3. Articulación del sistema de gestión integrado.

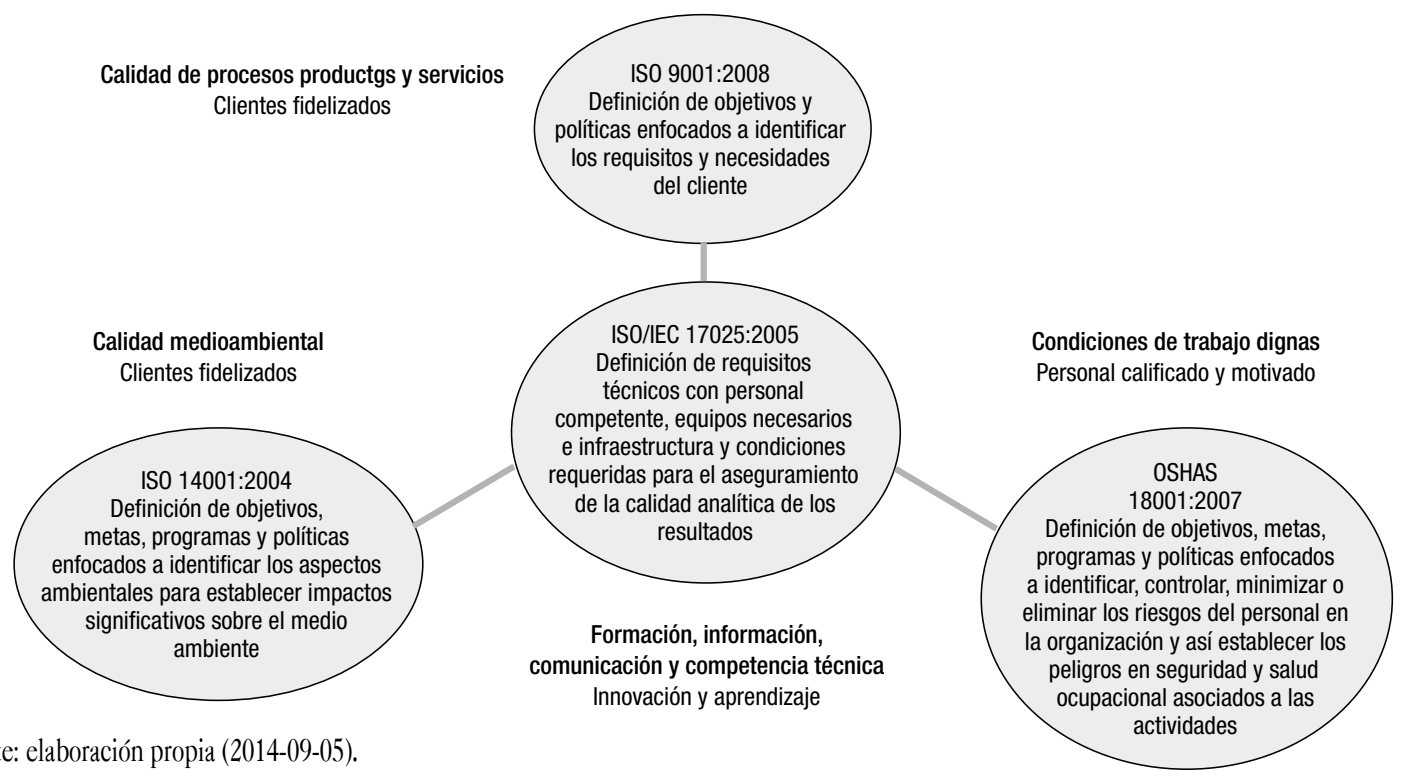


Nelcy Yamile Ortega Gómez

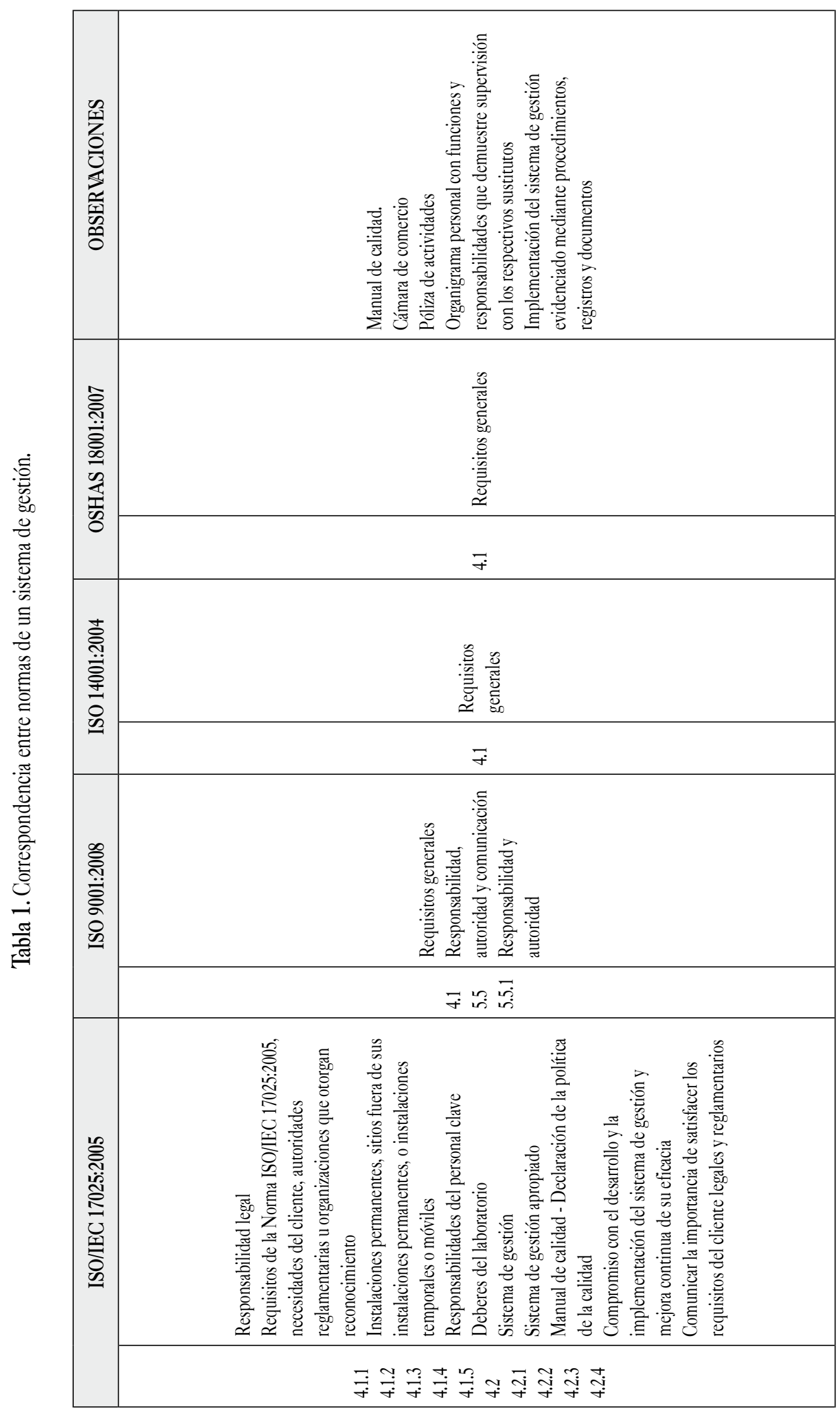




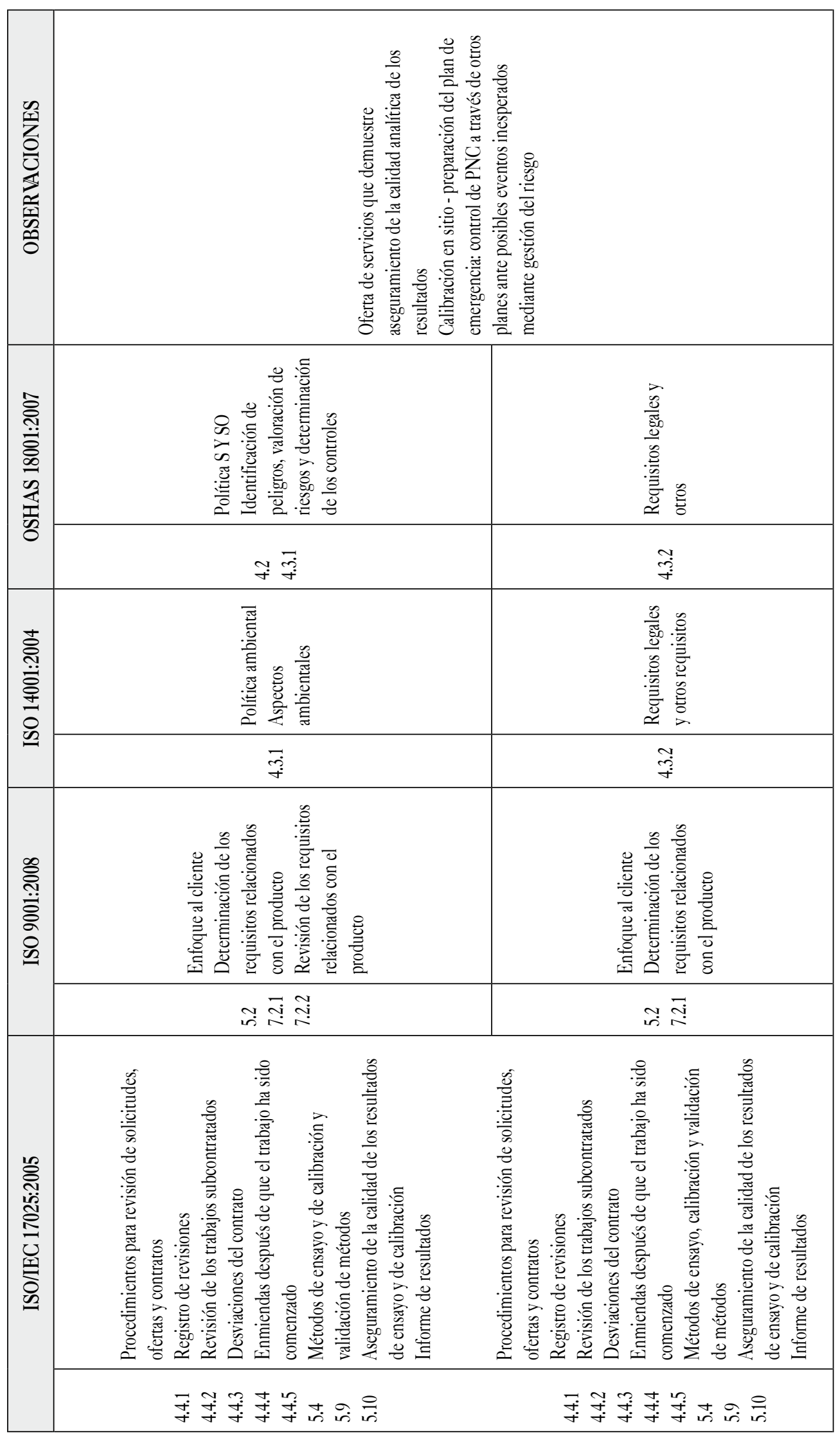




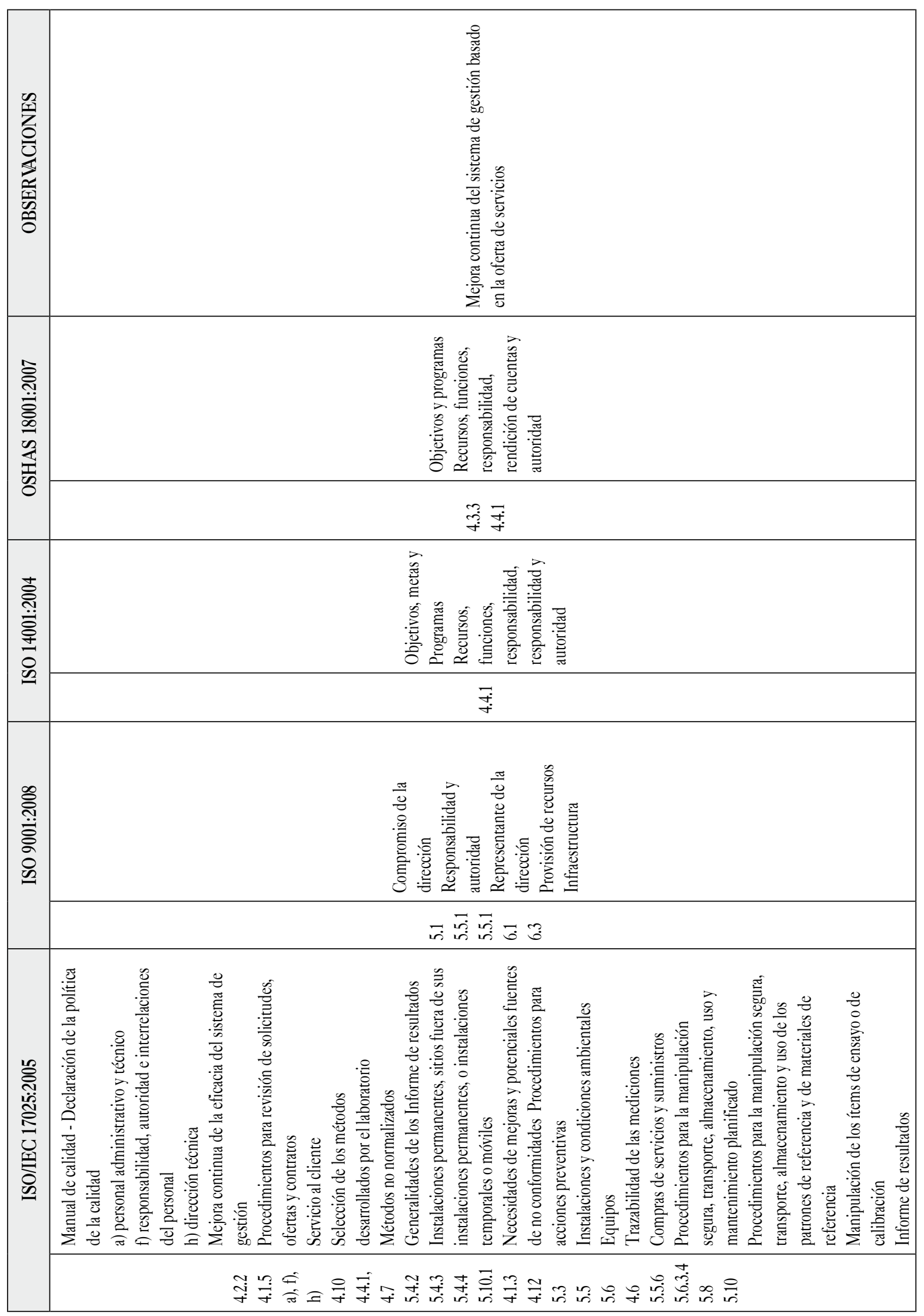




\begin{tabular}{|c|c|c|c|c|}
\hline 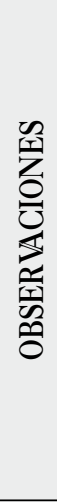 & 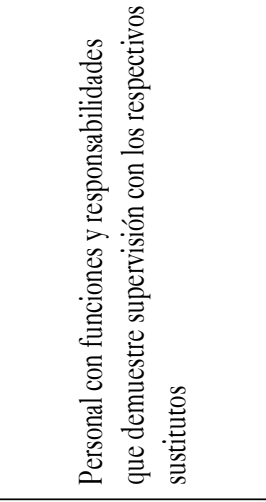 & 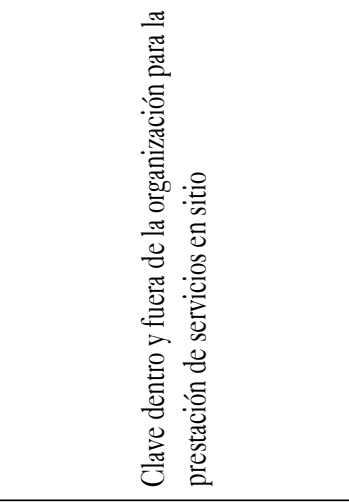 & \multicolumn{2}{|l|}{ 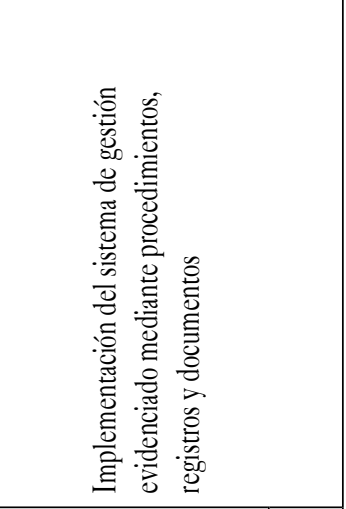 } \\
\hline \multirow[t]{2}{*}{ 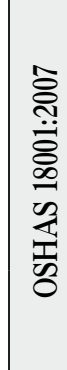 } & 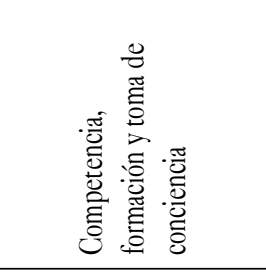 & 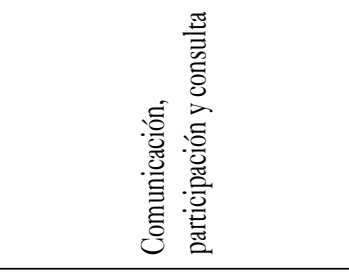 & 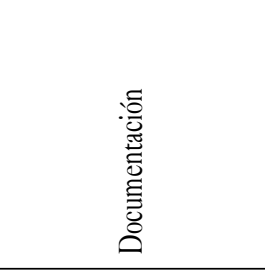 & 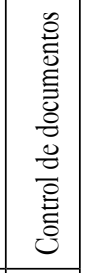 \\
\hline & 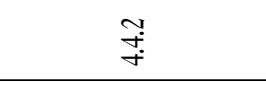 & $\stackrel{?}{+}$ & 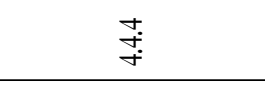 & 年 \\
\hline \multirow[t]{2}{*}{ 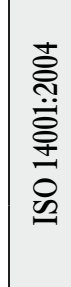 } & 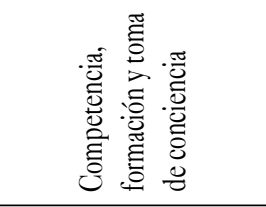 & 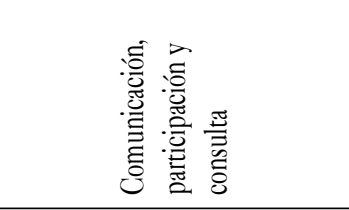 & 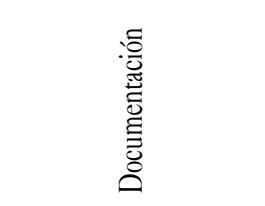 & 을 \\
\hline & $\stackrel{\stackrel{Y}{\ddagger}}{+}$ & $\stackrel{?}{+}$ & $\stackrel{\stackrel{+}{+}}{+}$ & $\stackrel{?}{\stackrel{2}{+}}$ \\
\hline \multirow[t]{2}{*}{ 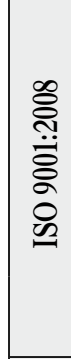 } & 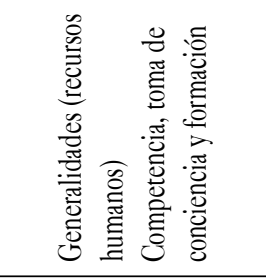 & 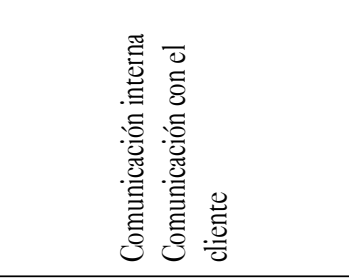 & 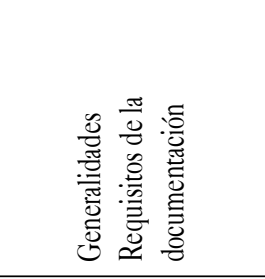 & 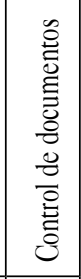 \\
\hline & $\overrightarrow{\mathcal{H}} \mathfrak{i}$ & กี่ & $\vec{i}$ & $\underset{\substack{i \\
+}}{+}$ \\
\hline \multirow[t]{2}{*}{ 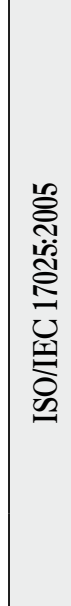 } & 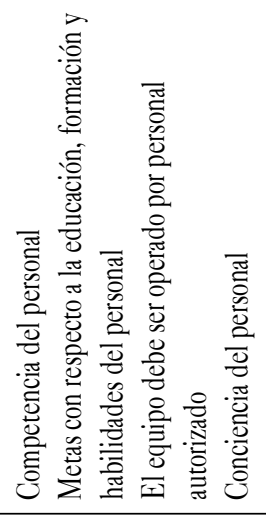 & 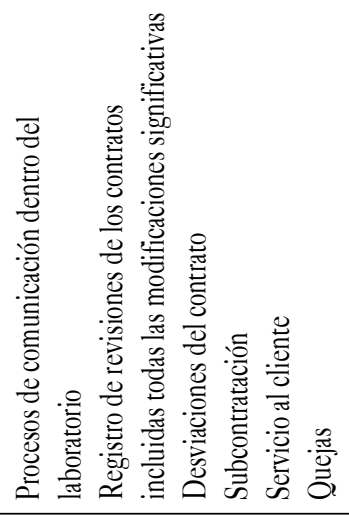 & 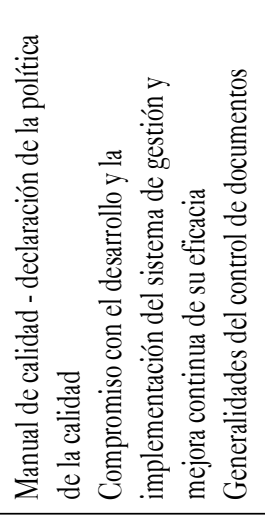 & 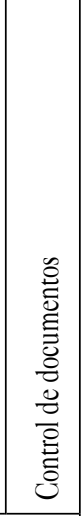 \\
\hline & 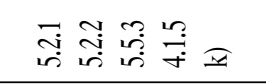 & 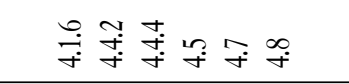 & 胥 & $\dddot{F}$ \\
\hline
\end{tabular}


Nelcy Yamile Ortega Gómez

\begin{tabular}{|c|c|}
\hline 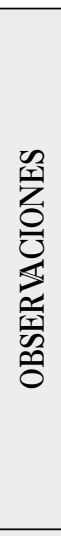 & 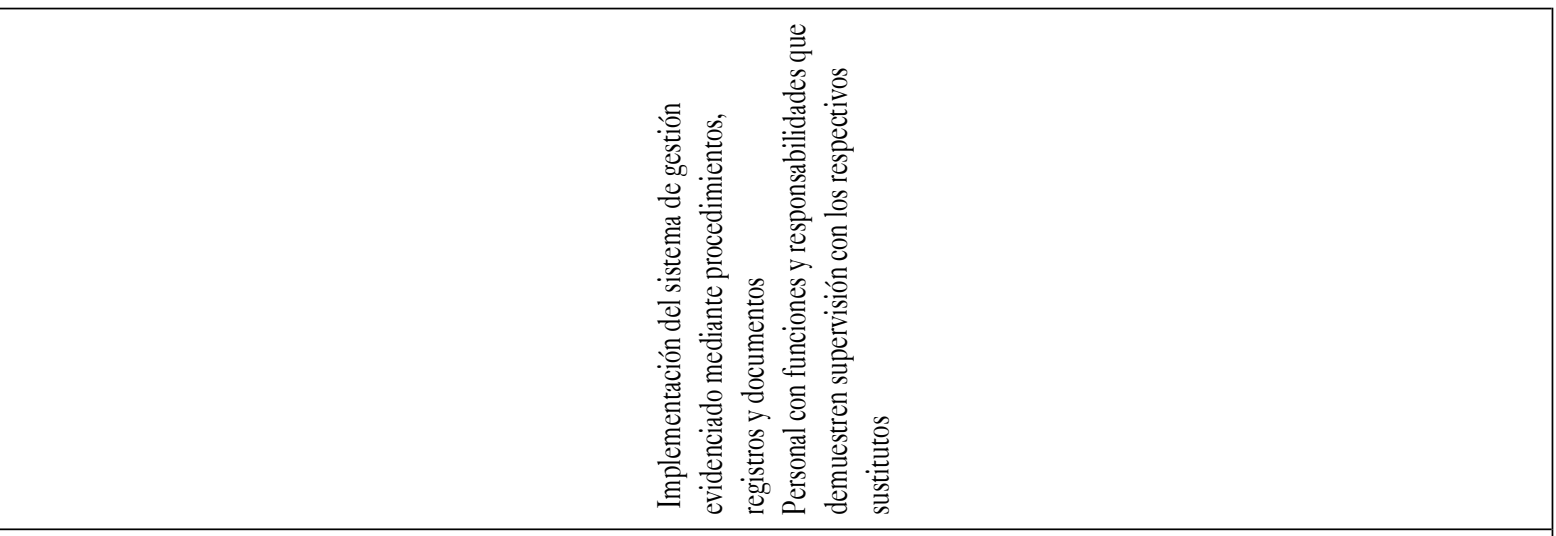 \\
\hline \multirow[t]{2}{*}{ 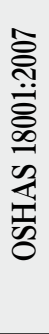 } & 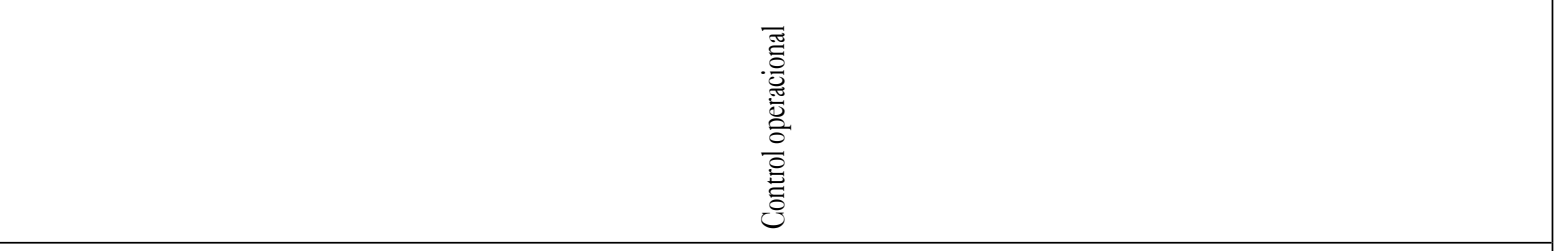 \\
\hline & $\stackrel{\substack{+0 \\
+}}{+}$ \\
\hline \multirow[t]{2}{*}{ 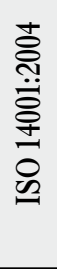 } & 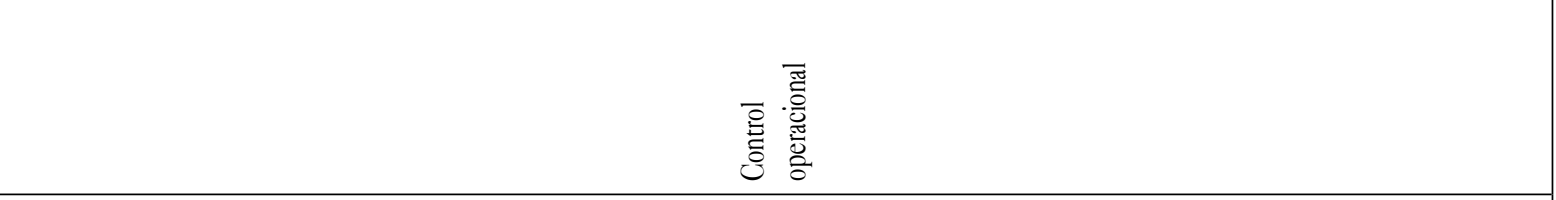 \\
\hline & $\stackrel{\substack{++}}{+}$ \\
\hline \multirow[t]{2}{*}{ 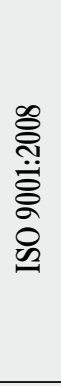 } & 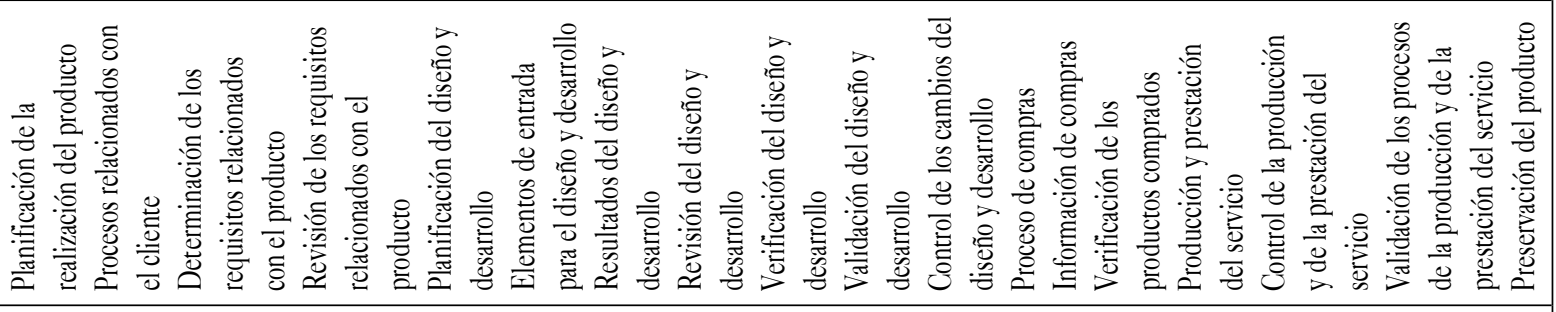 \\
\hline & 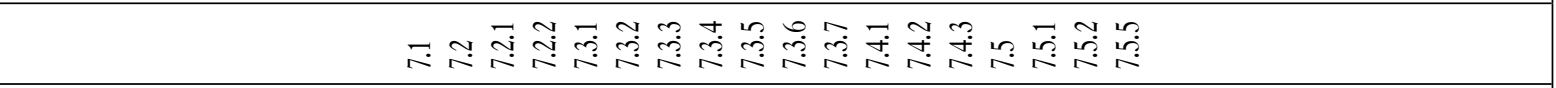 \\
\hline \multirow[t]{2}{*}{ 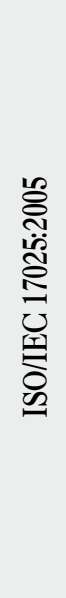 } & 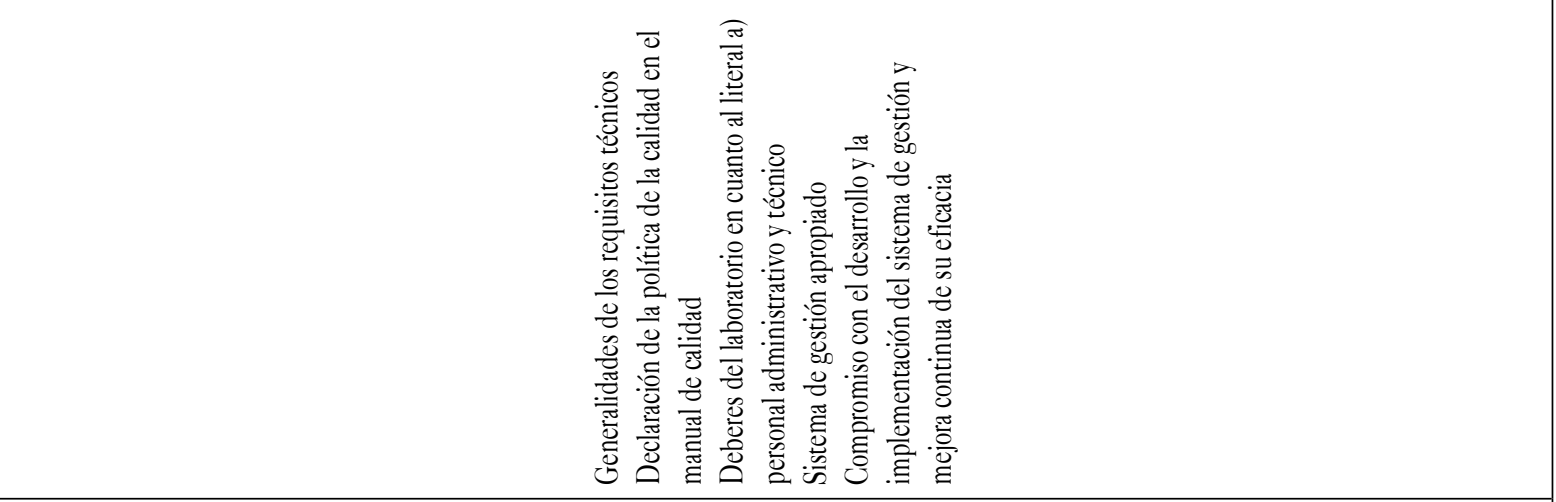 \\
\hline & 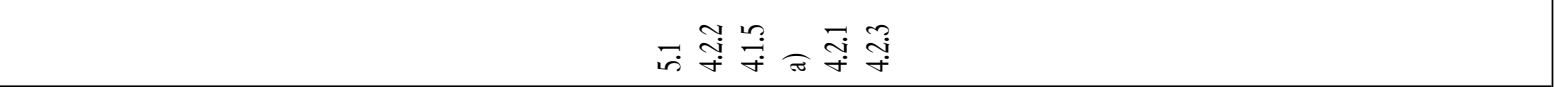 \\
\hline
\end{tabular}




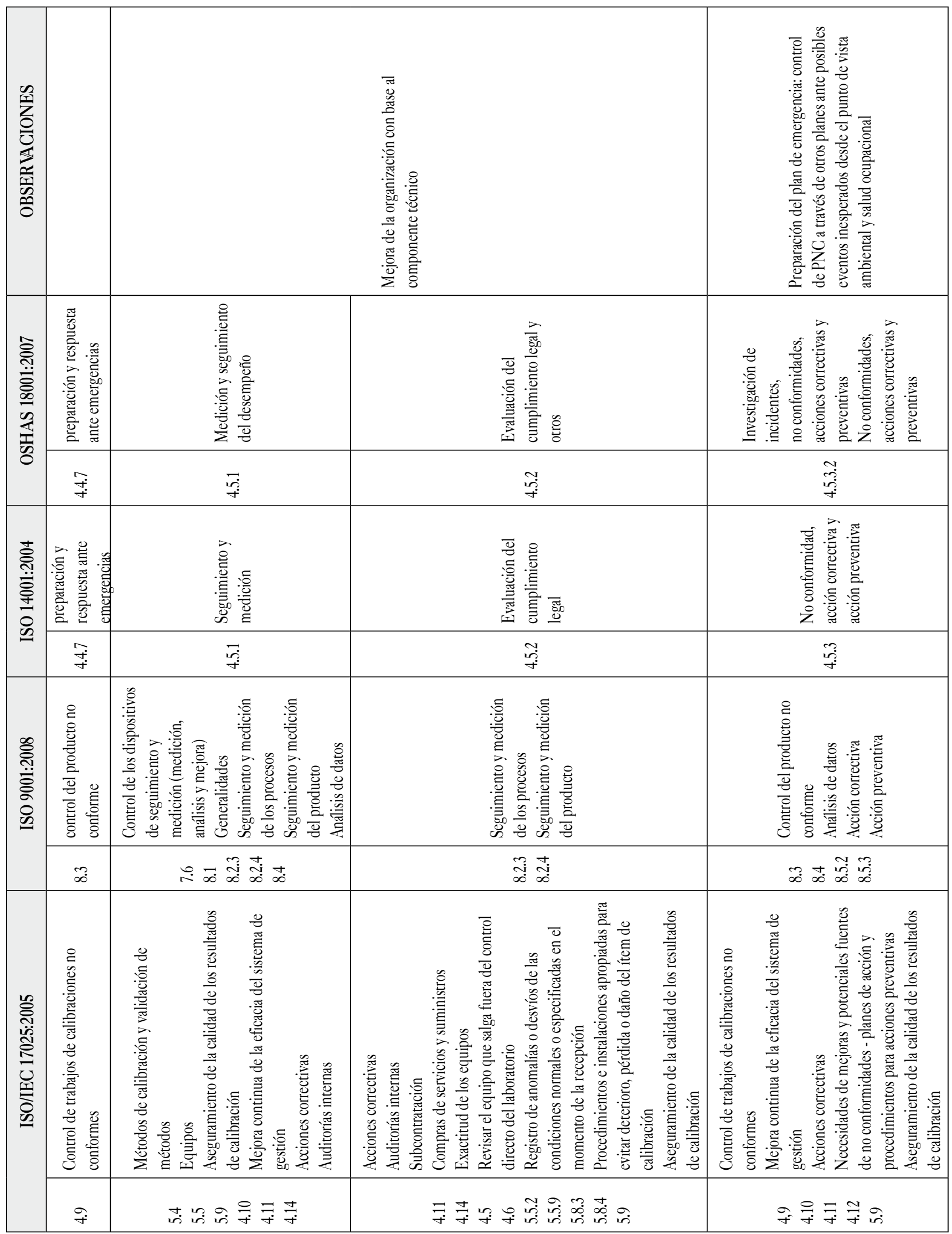


Nelcy Yamile Ortega Gómez

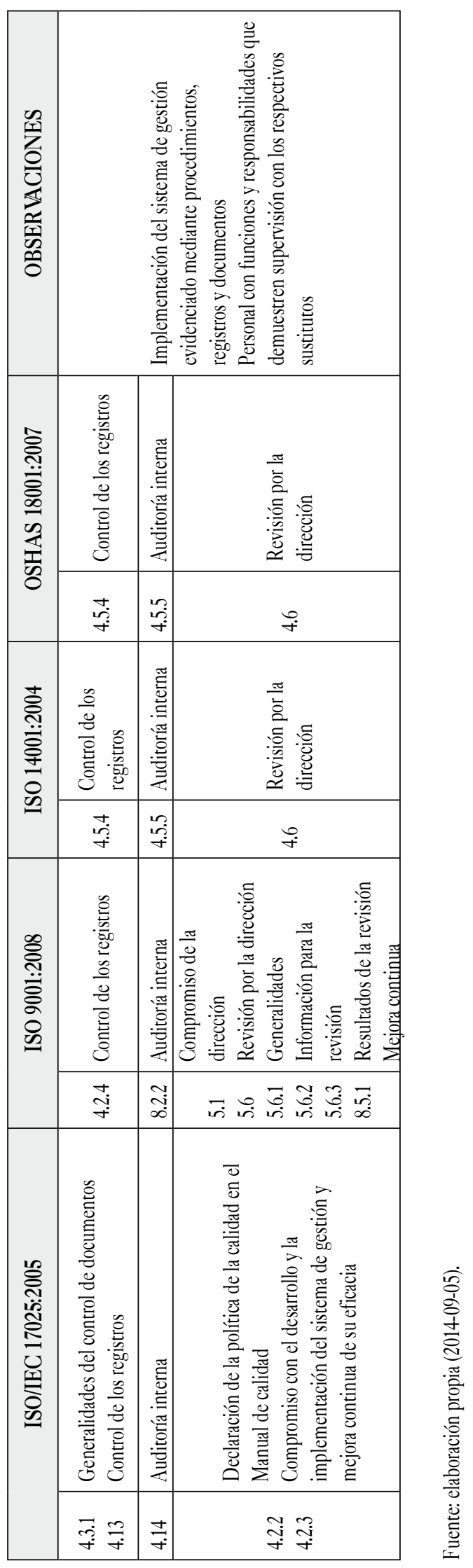




\section{RESULTADOS DE LAS ENCUESTAS - ANÁLISIS DESCRIPTIVO}

En la figura 4 se observa el porcentaje de participación de los OEC para responder la encuesta, razón por la cual la muestra fue del $83 \%$ de la población; de un total de 6 laboratorios acreditados en magnitudes químicas, cinco la respondieron. No fue fácil acceder a la realización de la encuesta, ya que los laboratorios exponían temas de confidencialidad porque la información que se solicitaba era muy técnica e iba direccionada al comportamiento de la organización frente a los servicios prestados, razón por la cual podría haber conflicto de intereses en cuanto a la competencia con otros laboratorios.

Los resultados obtenidos tuvieron una participación entre el 60 y el $100 \%$, máximo dos respuestas por laboratorio sin responder. Los laboratorios no se sentían seguros al responder tranquilamente todas las preguntas, pues consideraban que el enfoque de las encuestas los dejaba abiertamente expuestos a sus competidores en este tipo de servicios.
Figura 4. Participación de los OEC en la encuesta.

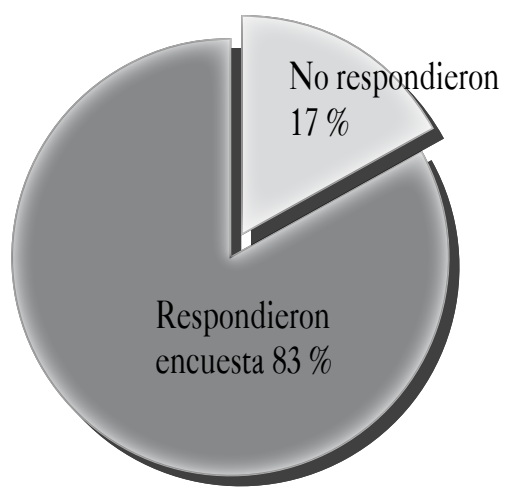

Fuente: elaboración propia (2014-09-05).

Todos los laboratorios han identificado sus clientes potenciales y consideran que estar acreditados o certificados mejora su nivel de competencia, es decir, el reconocimiento de un ente es importante para ellos, pero al profundizar en este tema no eran tan abiertos.

En la figura 5, el $60 \%$ de los laboratorios tienen la misma capacidad técnica instalada y de este el $20 \%$ la tiene subutilizada. Esto demuestra que están en la misma oportunidad de respuesta a sus clientes.

Figura 5. Capacidad del laboratorio.

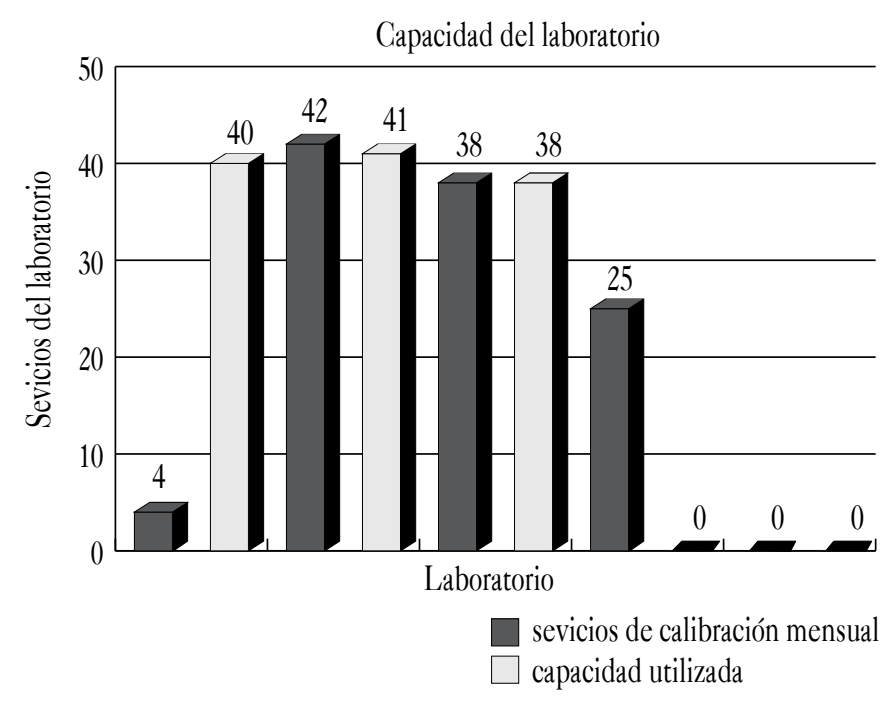

Fuente: elaboración propia (2014-09-05). 
Figura 6. Trayectoria de la organización.

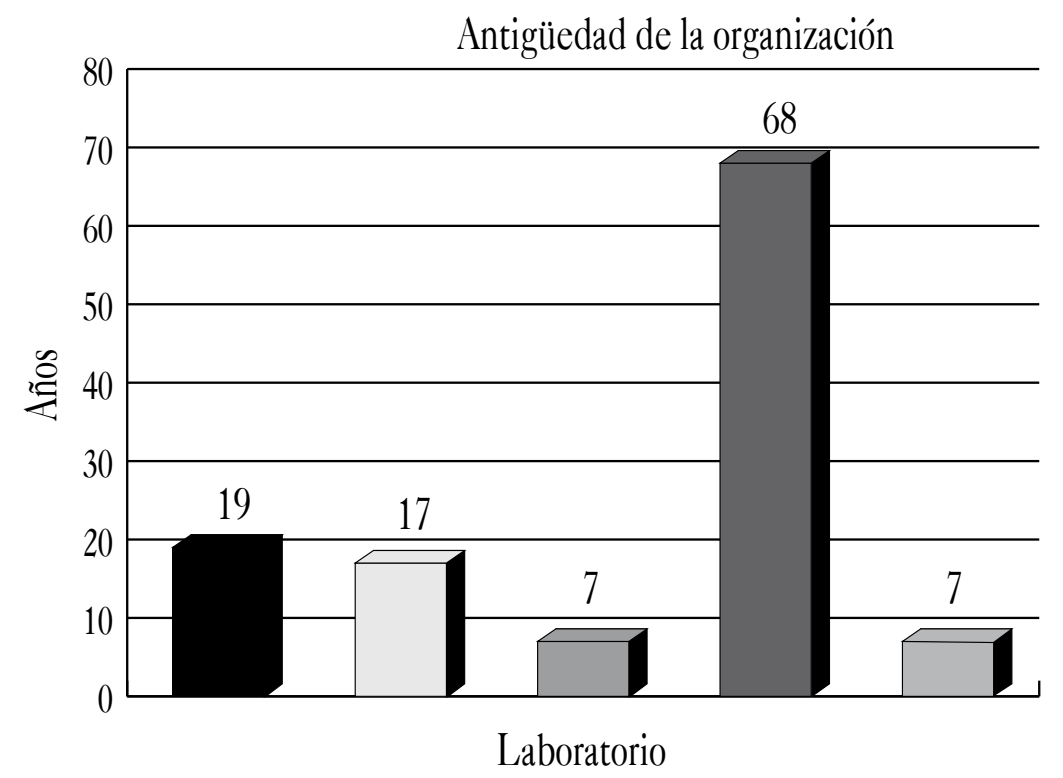

Fuente: elaboración propia (2014-09-05).

En la figura 6 se observa que las organizaciones a las que pertenecen estos laboratorios tienen entre 7 y 68 años de conformadas. Esto demuestra que son organizaciones sólidas, serias y estructuradas en el tiempo para prestar este servicio, pues solo dos laboratorios tienen menos de diez años de conformados y las otras tres entre 17 y 68 años.

En la tabla 2 se observa que el $100 \%$ manifestó que no tuvo TNC, el $60 \%$ no tuvo quejas en el mes anterior al desarrollo de la encuesta y el $100 \%$ de los encuestados expresan que sus clientes tienen un alto grado de satisfacción. Con estos resultados se demuestran que son organizaciones sólidas y competitivas técnicamente.

Tabla 2. Consolidación de resultados aspecto técnico.

\begin{tabular}{|l|c|}
\hline \multicolumn{2}{|c|}{$\begin{array}{c}\text { Componente técnico, } \\
100 \% \text { laboratorios }\end{array}$} \\
\hline TNC & No tiene \\
\hline Quejas & No tiene \\
\hline Satisfacción del cliente & Alto \\
\hline
\end{tabular}

En la tabla 3, se observa que el $20 \%$ de los laboratorios entrevistados tiene implementado además de la ISO/ IEC 17025:2005 la ISO 9001:2008, nada relacionado con la parte ambiental y salud ocupacional. El $20 \%$ no considera pertinente certificar sus procesos dentro de la organización, el $80 \%$ sí considera certificar el proceso técnico de calibración por calidad y confiabilidad, y de este último, el $20 \%$ considera importante también la gestión humana y la parte financiera. $\mathrm{Al}$ responder esta pregunta los laboratorios no tienen en cuenta otros sistemas de gestión, únicamente el de acreditación, no conocen ni tienen claridad sobre los sistemas de certificación, ni tienen en cuenta el componente ambiental ni de salud ocupacional, pues consideran que ese tema es únicamente requisito para los laboratorios de ensayos, sin tener en cuenta que la mayoría de ellos generan vertimientos durante sus calibraciones y el uso de sus patrones de calibración. Además de considerar aspectos relevantes de tipo ambiental y de salud ocupacional cuando realicen las calibraciones en sitio, es decir, en las instalaciones del cliente.

Fuente: elaboración propia (2014-09-05). 
Importante que los laboratorios conozcan que con la implementación de la ISO/IEC 17025:2005 ya dan cumplimiento a otras normas tales como la NTC-ISO 9001:2008, NTC-OHSAS 18001:2007, NTC-ISO 14001:2004; solo que deben tener claridad en los requerimientos de cada una. Esto les puede ampliar su espectro de servicios y llegar a los clientes potenciales para mejorar su capacidad técnica del momento.

Tabla 3. Consolidación de resultados alta dirección.

\begin{tabular}{|l|c|}
\hline \multicolumn{2}{|c|}{ Componente alta dirección } \\
\hline Ha identificado clientes potenciales & $100 \%$ \\
\hline Acreditación/certificación mejora la competencia técnica & $100 \%$ \\
\hline Pertinente certificar sus procesos & $80 \%$ \\
\hline Conocer certificación de otros OEC del sector & $60 \%$ \\
\hline $\begin{array}{l}\text { Tiene implementado otro sistema de gestión a la ISO/ } \\
\text { IEC 17025:2005 }\end{array}$ & $20 \%$ \\
\hline
\end{tabular}

Fuente: elaboración propia (2014-09-05).

\section{CONCLUSIONES}

La matriz teórica elaborada permitió establecer la articulación, de acuerdo con los requisitos normativos establecidos, entre las normas técnicas NTC-ISO 9001:2008, NTC-OHSAS 18001:2007, NTC-ISO 14001:2004 y NTC-ISO/IEC 17025:2005, a partir de la acreditación de los laboratorios, es decir desde el punto de vista del componente técnico, de infraestructura y equipos; teniendo en cuenta que los requisitos de un sistema de gestión se desarrollan bajo el ciclo PHVA.

A partir de la matriz teórica construida y de indicadores de gestión tales como trabajo no conforme, quejas y encuestas de satisfacción se elabora la encuesta que permite identificar los siguientes aspectos de los sistemas de gestión enmarcados en un ciclo PHVA para medir la optimización de recursos en términos de eficacia (resultados) y eficiencia (procesos) en los laboratorios de calibración acreditados base de este estudio:
- Conocimiento del contexto de las actividades del personal y de la organización.

- Interacción con otras áreas de la industria y la academia para aumentar la capacidad instalada.

- Reducir costos en implementación de los sistemas de gestión de acreditación y certificación.

- Fortalecer la organización en los conceptos de sistemas de gestión para enfrentar otras plazas de mercados.

- Se identificó que los sistemas de gestión implementados en los laboratorios de calibración bajo la NTC-ISO/IEC 17025:2005 y la NTC-ISO 9001:2008 solo buscan la confiabilidad de los resultados y mantenerse en un sector especializado, pero no hay conocimiento ni interés en implementar la NTC-OHSAS 18001:2007 y la NTC-ISO 14001:2004.

Frente al concepto de globalización (Stiglitz, 2006) se puede confirmar que a través de los laboratorios acreditados con ONAC, Colombia comienza a participar en el ámbito internacional con una actividad técnicaeconómica que demuestra el compromiso del gobierno y de la industria para competir, con herramientas como la implementación del sistema de gestión mediante normas internacionales para la calidad, que garantizan que los servicios que ofrecen estos laboratorios son creibles y fiables.

Para que los laboratorios con alcances acreditados con ONAC continúen con el fomento del comercio y el desarrollo en este campo de acción de servicios tan amplio, deben seguir fortaleciendo su sistema de gestión con un enfoque sistémico, oportuno y estructurado y apalancado en las redes intersectoriales disponibles hoy en día en el país.

Se evidencia la necesidad de reforzar la formación en los conceptos y la relación entre calidad, certificación y 
acreditación, en el personal a cargo de los sistemas de gestión de las organizaciones, para fortalecer la competitividad y el reconocimiento internacional de este sector.

La ejecución de actividades técnicas de laboratorio que demuestren una calidad medioambiental, que cuenten con personal calificado y motivado en condiciones de trabajo dignas, conducen a la fidelización de los clientes y traen nuevas oportunidades de negocio en una Colombia globalizada que ya cuenta con laboratorios acreditados y con reconocimiento internacional.

\section{REFERENCIAS}

Canavos, J. C. (1988). Probabilidad y estadística. Aplicaciones y métodos. México: McGraw-Hill Interamericana de México S. A.

Cerda, H. (2011). Los elementos de la investigación: cómo reconocerlos, diseñarlos y construirlos. Bogotá: Editorial Magisterio.

Instituto Colombiano de Normas Técnicas y Certificación - Icontec. (2008). NTC-ISO 9001. Sistema de gestión de la calidad. Requisitos. Bogotá D. C.: Icontec.

Instituto Colombiano de Normas Técnicas y Certificación - Icontec. (2004). NTC-ISO 14001. Sistemas de gestión ambiental. Requisitos con orientación para su uso. Bogotá D. C.: Icontec.
Instituto Colombiano de Normas Técnicas y Certificación - Icontec. (2005). NTC-ISO/IEC17011. Evaluación de la conformidad. Requisitos generales para los organismos de acreditación que realizan la acreditación de organismos de evaluación de la conformidad. Bogotá D. C.: Icontec.

Instituto Colombiano de Normas Técnicas y Certificación - Icontec. (2005). NTC-ISO/IEC17025.Requisitos generales para la competencia de los laboratorios de ensayo y calibración. Bogotá D. C.: Icontec.

Instituto Colombiano de Normas Técnicas y Certificación - Icontec. (2007). NTC-OHSAS 18001. Sistemas de gestión en seguridad y salud ocupacional. Requisitos. Bogotá D. C.: Icontec.

Presidencia de la República. (1993, 16 de noviembre). Decreto 2269. Por el cual se organiza el sistema nacional de normalización, certificación y metrología. Diario Oficial 41110. Bogotá, D. C.

Stiglitz, J. E. (2006, noviembre). Cómo hacer que funcione la globalización. Madrid: Taurus.

Superintendencia de Industria y Comercio. (2006). Sistema nacional de normalización, certificación y metrología base para el fortalecimiento de la competitividad. Pereira. Recuperado de http://www. sic.gov.co/recursos_user/documentos/articulos/ SistemaNacionalNormalizacion.pdf 